\begin{abstract}
Studien und Materialien zum Straf- und Maßregelvollzug

herausgegeben von

Friedrich Lösel, Gerhard Rehn und Michael Walter
\end{abstract}

BAND 24 


\section{Psychologische Schuldfähigkeitsbegutachtung bei sogenannter schwerer anderer seelischer Abartigkeit}

Eine Begutachtungsheuristik
auf empirischer Grundlage

Alexander F. Schmidt



Centaurus Verlag \& Media UG 2008 
Alexander F. Schmidt, geb. 1973, Dr. phil., Dipl.-Psych., ist wissenschaftlicher Mitarbeiter am Institut für Psychologie der Universität Bonn, Abteilung Sozial- und Rechtspsychologie. Psychologischer Psychotherapeut mit Schwerpunkt Verhaltenstherapie in Ausbildung, langjähriger wissenschaftlicher Mitarbeiter am ehemaligen Lehrstuhl für Klinische und Angewandte Psychologie der Universität Bonn. Publikationstätigkeit insbesondere zum Thema Schuldfähigkeitsbegutachtung, zu Straftäterbehandlung, zu Dissozialität sowie zu Emotionsregulation und Psychotherapie.

Die Deutsche Bibliothek - CIP-Einheitsaufnahme

\section{Schmidt, Alexander F.:}

Begutachtung der Schuldfähigkeit bei sogenannter schwerer anderer seelischer Abartigkeit / Alexander F. Schmidt.-

Herbolzheim: Centaurus-Verl., 2008

(Studien und Materialien zum Straf- und Maßregelvollzug; Bd. 24)

Zugl.: Bonn, Univ., Diss., 2007

ISBN 978-3-8255-0676-6 ISBN 978-3-86226-450-6 (eBook)

DOI 10.1007/978-3-86226-450-6

\section{ISSN 0944-887X}

Alle Rechte, insbesondere das Recht der Vervielfältigung und Verbreitung sowie der Übersetzung, vorbehalten. Kein Teil des Werkes darf in irgendeiner Form (durch Fotokopie, Mikrofilm oder ein anderes Verfahren) ohne schriftliche Genehmigung des Verlages reproduziert oder unter Verwendung elektronischer Systeme verarbeitet, vervielfältigt oder verbreitet werden.

(c) CENTAURUS Verlags-GmbH \& Co. KG, Herbolzheim 2008

Satz: Vorlage des Autors

Umschlaggestaltung: Antje Walter, Titisee 
Die Gefahr, als jemand etikettiert zu werden, der die „Wahrheit“ kennt, schränkt sowohl den „Experten“ als auch diejenigen, die vom „Experten“ lernen möchten, ein.

(Cecchin, Lane \& Ray, 1996, S.18) 


\section{Danksagung}

Keine Forschungsarbeit entsteht losgelöst von ihren Rahmenbedingungen. An dieser Stelle soll allen Personen herzlich gedankt werden, die diese Untersuchung in der vorliegenden Form überhaupt erst möglich gemacht und mit ihrem Bemühen um deren Ergebnisse zum Besseren beigetragen haben. Mein besonderer Dank gilt dabei meinen Eltern und meiner Familie, die mir maßgebliche Erfahrungen ermöglichten, welche schlussendlich zu dieser Untersuchung geführt haben.

Ferner möchte ich mich bei meinem Erstgutachter und Betreuer Prof. Dr. O. Berndt Scholz, der die Realisierung dieses Forschungsprojekts unter Gewährung von großen Freiheiten begleitet hat, für die langjährige Unterstützung bedanken. Mein Dank gilt auch Prof. Dr. Nobert Nedopil, ohne dessen großzügige Kooperation die Untersuchung in dieser Form nicht zustande gekommen wäre, und der stets ein Forum schuf, Zwischenergebnisse berichten und diskutieren zu können.

Zwingend notwendige Arbeiten nur bedingt reizvoller Natur sind Korrekturen, denen Dipl.-Psych. Clarissa Leipert ihre wertvollen Ressourcen geopfert hat. Ferner stand sie mir auch in Zeiten größter Belastung stets zur Seite und hat damit einen unschätzbaren Anteil an den Rahmenbedingungen mitgetragen, die für die Abfassung dieser Arbeit hilfreich und notwendig waren. Auch meinem lieben Kollegen Dr. Frank Kaspers sei an dieser Stelle für die humorvolle Begleitung im Forschungsalltag gedankt. Unbedingt zu erwähnen ist auch Dr. Andreas Mokros, der einen wichtigen Impuls gab.

Schließlich verbindet sich mein Dank auch mit meiner Zweitgutachterin Prof. Dr. Marie-Luise Kluck und Prof. Dr. Rainer Banse, der im richtigen Moment Freiheiten und Möglichkeiten gewährte, dieses Forschungsprojekt zu seinem Ende zu bringen.

Bonn im Dezember 2007

Alexander F. Schmidt 


\section{Inhalt}

\section{RECHTLICHE GRUNDLAGEN UND MINDESTANFORDERUNGEN DER}

SCHULDFÄHIGKEITSBEGUTACHTUNG .5

2.1 Schuldbegriff und die Rolle der Willensfreiheit im deutschen Strafrecht............................................5

2.2 Die Bestimmungen zur Schuldfähigkeit im deutschen Strafrecht ......................................................8

2.2.1 Die erste Stufe der Schuldfähigkeitsbegutachtung - Eingangsmerkmale und Krankheitsbegriff........10

2.2.2 Die zweite Stufe der Schuldfähigkeitsbegutachtung - Auswirkungen auf die Einsichts- und

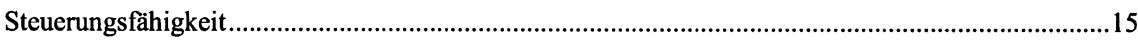

2.3 Mindestanforderungen für Schuldfähigkeitsgutachten aus juristischer Sicht.......................................18

3 DIE SOGENANNTE SCHWERE ANDERE SEELISCHE ABARTIGKEIT AUS PSYCHIATRISCH-PSYCHOLOGISCHER SICHT UND MINDESTSTANDARDS FÜR DIE BEGUTACHTUNG

3.1 Die Begrifflichkeit der sogenannten schweren anderen seelischen Abartigkeit. .22

3.2 Zentrale Lösungsansätze der Schuldfähigkeitsbegutachtung bei sogenannter schwerer anderer seelischer Abartigkeit .27

3.2.1 Der Hamburger Ansatz der progredienten psychopathologischen Entwicklung nach Schorsch .........27

3.2.2 Tiefenpsychologische Ansätze nach Mundt und Böhle ...................................................................30

3.2.3 Der Tübinger Ansatz zur Quantifizierung nach Foerster.............................................................32

3.2.4 Der Berliner Ansatz des strukturell-sozialen Krankheitsbegriffs nach Rasch.....................................34

3.2.5 Der Aachener Ansatz des psychopathologischen Referenzsystems und die Kriterienliste von $\mathrm{SaB}$.

3.2.6 Der Bonner Ansatz der Schuldfähigkeitskategorien von Scholz und Schmidt ................................40

3.3 Mindestanforderungen der Begutachtung von sogenannter schwerer anderer seelischer Abartigkeit aus psychologisch-psychiatrischer Sicht .42

3.3.1 Mindeststandards der Begutachtung von Persönlichkeitsstörungen.................................................44

3.3.2 Mindeststandards der Begutachtung von Sexualdelinquenz...........................................................46

4 PSYCHOLOGISCHE AUSWIRKUNGEN SOGENANNTER SCHWERER ANDERER SEELISCHER ABARTIGKEIT

4.1 Makroebene der Schuldfähigkeitsbegutachtung - auf dem Weg zu einer Rahmentheorie der

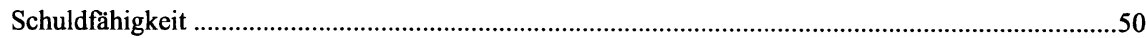

4.1.1 Das Vulnerabilitäts-Stress-Modell als allgemeine Konzeption psychischer Störungen.......................51

4.1.2 Seelische Gesundheit und Verhaltenskontrolle im Kontext der Schuldfähigkeit.................................53

4.2 Mikroebene der Schuldfähigkeitsbegutachtung - Situationsübergreifende Erlebens- und Verhaltensdispositionen der ersten Schuldfähigkeitsachse

4.2.1 Problemlösen, Emotionsregulation, Abwehrmechanismen und Schuldfähigkeit................................63

4.2.1.1 Die Struktur-Achse der Operationalisierten Psychodynamischen Diagnostik OPD ...........................71

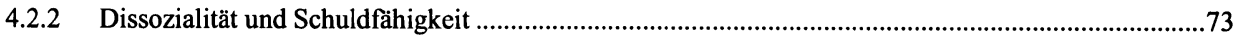


4.2.2.1 Das Persönlichkeitskonstrukt der Psychopathy nach Hare .7

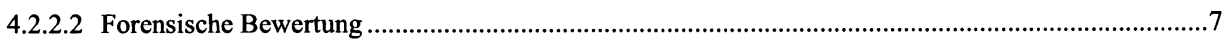

4.3 Mikroebene der Schuldfähigkeitsbegutachtung - Tatbezogene Erlebens- und Verhaltensweisen der zweiten Schuldfähigkeitsachse ................................................................. 8

4.3.1 Zeitliche und handlungstheoretische Aspekte des Tatverhaltens ....................................................8

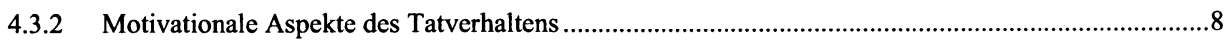

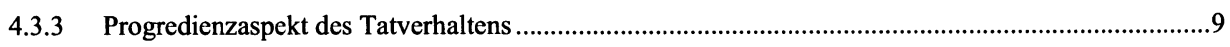

4.4 Von der Makro- zur Mikroebene der Schuldfähigkeitsbegutachtung - eine Zusammenfassung.........9

5 ENTWICKLUNG EMPIRISCH BASIERTER SCHULDFÄHIGKEITSMERKMALE DER SOGENANNTEN SCHWEREN ANDEREN SEELISCHEN ABARTIGKEIT ..................................9

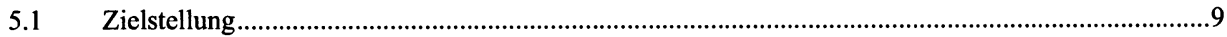

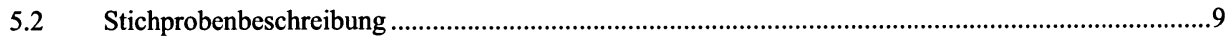

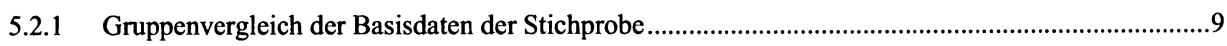

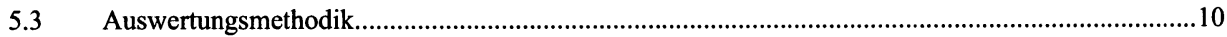

5.3.1 Qualitative Analyse - Skalierende Strukturierung...................................................................10

5.3.1.1 Kategoriensystem - Kurzbeschreibung der Indikatoren der Schuldfähigkeitsachse zu situationsübergreifenden Erlebens- und Verhaltensdispositionen ...................................................10

5.3.1.2 Kategoriensystem - Kurzbeschreibung der Indikatoren der Schuldfähigkeitsachse Tatbezogene Erlebens- und Verhaltensweisen

5.3.1.3 Kategoriensystem - Kurzbeschreibung der Indikatoren zur Erfassung der Persönlichkeitsfaktoren Seelische Gesundheit und Verhaltenskontrolle...........................................11

5.3.2 Quantitative Analyse - Aggregation zu Schuldfähigkeitskategorien ...............................................11

5.3.3 Quantitative Analyse - Überprüfung der Diskriminations- und Klassifikationsleistung anhand binär logistischer Regressionen und ROC-Analysen..........................................................................11

5.3.4 Analyse der Fehlklassifikationen ............................................................................................11

5.3.5 Überprüfung des Zusammenhangs der Persönlichkeitsfaktoren Seelische Gesundheit und Verhaltenskontrolle mit den Schuldfähigkeitsempfehlungen.

5.4 Empirisch basierte Schuldfähigkeitsmerkmale - die Ergebnisse der quantitativen

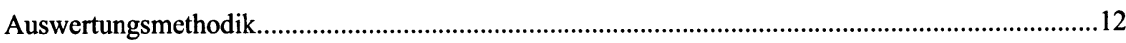

5.4.1 Aggregation von Schuldfähigkeitskategorien .................................................................................12

5.4.1.1 Schuldfähigkeitskategorien der Achse I - Situationsübergreifende Erlebens- und

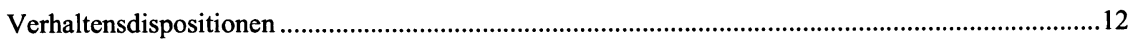

5.4.1.2 Schuldfähigkeitskategorien der Achse II - Tatbezogene Erlebens- und Verhaltensweisen...............12

5.4.2 Replizierbarkeit der Schuldfähigkeitskategorien ..............................................................................12

5.4.3 Psychometrische Kennwerte der Schuldfähigkeitskategorien beider Achsen ..................................13

5.4.4 Die Diskriminations- und Klassifikationsleistung der Schuldfähigkeitsmerkmale ...........................13

5.4.4.1 Trennschärfe der Achse I - Situationsübergreifende Erlebens- und Verhaltensdispositionen............13

5.4.4.2 Trennschärfe der Achse II - Tatbezogene Erlebens- und Verhaltensweisen .....................................13

5.4.5 Minimalkonfiguration von Schuldfähigkeitsmerkmalen der Achsen I und II..................................14

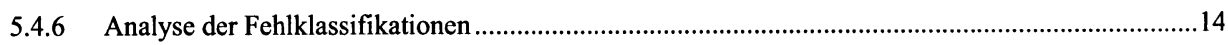


5.4.7 Die Persönlichkeitsfaktoren Seelische Gesundheit und Verhaltenskontrolle im Verhältnis zur Schuldfähigkeitseinschätzung durch die Sachverständigen.

6.1 Die Bedeutung der empirisch basierten Schuldfähigkeitsmerkmale für die

Schuldfähigkeitsfrage

6.1.1 Situationsübergreifende Erlebens- und Verhaltensdispositionen - die erste Schuldfähigkeitsachse

6.1.1.1 Passiv-vermeidendes vs. aktiv-adaptives Problemlösen..................................................................153

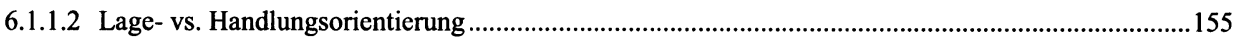

6.1.1.3 Impulsiv-explosibles Verhalten vs. Impulskontrolle und Selbststeuerung........................................156

6.1.1.4 Externalisierend-egozentrische vs. prosozial-reziproke Affektivität..................................................158

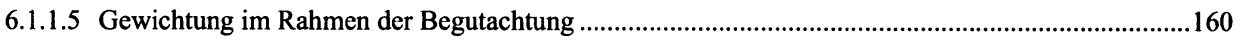

6.1.1.6 Zur Rolle der Dissozialität für die Schuldfähigkeitsbegutachtung ..................................................164

6.1.2 Tatbezogene Erlebens- und Verhaltensweisen - die zweite Schuldfähigkeitsachse ...........................166

6.1.2.1 Labilisierung vs. emotionale Stabilität im Vorfeld der Tat ...........................................................166

6.1.2.2 Affektregulativ-egodystone vs. instrumentell-egosyntone Tatbegehung ..........................................168

6.1.2.3 Progredientes vs. nicht-progredientes Tatverhalten....................................................................169

6.1.2.4 Gewichtung im Rahmen der Begutachtung ................................................................................171

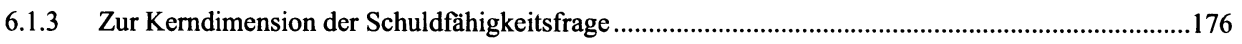

6.2 Schuldfähigkeit als stressbewältigendes Handeln im Vulnerabilitäts-Stress-Modell.........................178

6.3 Eine integrative Heuristik der Schuldfähigkeitsbegutachtung bei sogenannter schwerer anderer

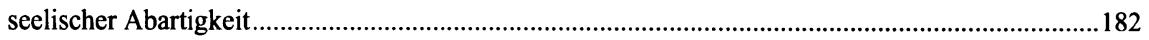

6.4 Methodenkritik, offene Fragen und zukünftige Forschungsdesiderate..........................................187

ANHANG A - OPERATIONALISIERTER KODIERLEITFADEN FÜR DIE SCHULDFÄHIGKEITSACHSEN

Achse I : Situationsübergreifende Erlebens- und Verhaltensdispositionen - Trait-Achse ..............................206

Achse II: Tatbezogene Erlebens- und Verhaltensweisen - State-Achse ....................................................213

ANHANG B - OPERATIONALISIERUNGEN FÜR DIE PERSÖNLICHKEITSFAKTOREN SEELISCHE GESUNDHEIT UND VERHALTENSKONTROLLE NACH BECKER (1995).......219

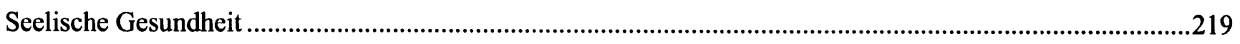

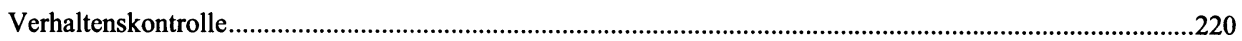

ANHANG C - INTERKORRELATIONSMATRIX DER SCHULDFÄHIGKEITSKATEGORIEN UND ÜBERSICHT DER VARIABLENZUORDNUNGEN 